\title{
Optimized Beam Optics for Muon Acceleration
}

\author{
S.A. Bogacz \\ Center for Advanced Studies of Accelerators \\ Thomas Jefferson National accelerator facility \\ Newport News, VA 23606
}

\begin{abstract}
A conceptual design of a Ring Cooler driven muon acceleration based on recirculating superconducting linacs is proposed. In the presented scenario, acceleration starts after ionization cooling at 250 $\mathrm{MeV} / \mathrm{c}$ and proceeds to $20 \mathrm{GeV}$, where the beam is injected into a neutrino factory storage ring. The key technical issues are addressed; such as: the choice of acceleration technology (superconducting versus normal conducting) and the choice of RF frequency, and finally, implementation of the overall acceleration scheme: capture, acceleration, transport and preservation of large phase space of fast decaying species. Beam transport issues for large-momentum-spread beams are accommodated by appropriate lattice design choices. The proposed arc optics is further optimized with a sextupole correction to suppress chromatic effects contributing to emittance dilution.
\end{abstract}

\section{MUON ACCELERATION SCHEME}

A neutrino factory [1] is aimed to produce narrow neutrino beams via decay of muons in long straight sections of a storage ring. Recent development of Ring Coolers promises much larger longitudinal phase space compression compared to previously considered straight Cooling Channels. Here we propose an optimized muon accelerator complex based on a Ring Cooler as a front end. As illustrated schematically in Figure 1, a proposed muon accelerator features a 250 MeV-to-2.8 GeV straight pre-accelerator linac and a 2.8-to-20 GeV four-pass recirculating linac accelerator (RLA).

The pre-accelerator captures a large muon phase space coming from the Ring Cooler and accelerates muons to relativistic energies of about $2.8 \mathrm{GeV}$. It makes the beam sufficiently relativistic and adiabatically decreases the phase-space volume, so that effective acceleration in recirculating linacs is possible. The RLA further compresses and shapes the longitudinal and transverse phase spaces, while increasing the energy.

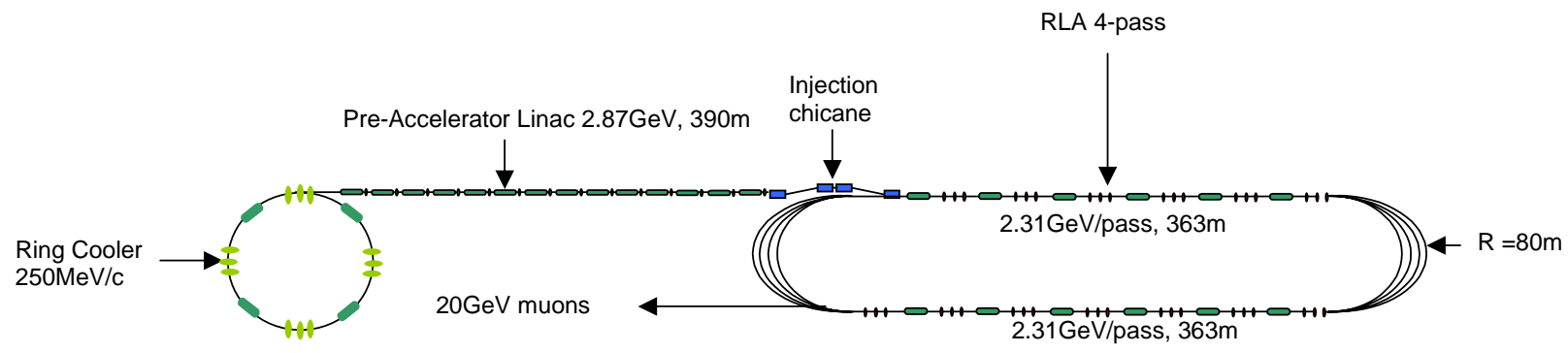

FIGURE 1. Ring Cooler driven design of a $20 \mathrm{GeV}$ muon accelerator complex based on RLA - machine layout. 


\section{Accelerating Technology}

To ensure adequate survival rates of short-lived muons, acceleration must occur at high average gradient. Initial estimate [2] shows that a "real estate average" RF gradient of $15 \mathrm{MV} / \mathrm{m}$ will allow survival of about $80 \%$ of source muons throughout the RLA. Since muons are generated as a secondary beam they occupy large phase-space volume. The accelerator must provide high average gradient, while maintaining very large transverse and longitudinal accelerator acceptances. The above requirement drives the design to low RF frequency, e.g. $200 \mathrm{MHz}$. If normalconducting cavities at that frequency were used, the required high gradients would demand unachievably high peak RF sources. The RF power can then be delivered to the cavities over an extended time, and thus RF source peak power can be reduced.

\section{Machine Architecture}

In a recirculating linac accelerator one needs to separate different energy beams coming out of a linac and to direct them into appropriate arcs for recirculation. For multiple practical reasons horizontal rather than vertical beam separation was chosen [2]. Rather than suppressing horizontal dispersion created by the spreaders and recombiners it is smoothly matched to the horizontal dispersion of the arc. Finally, to assure compact arc architecture very short matching sections in spreaders and recombiners are desired. One also needs to maintain manageable beam sizes in the arcs [3]. This calls for short cells and for putting stringent limits on dispersion and beta functions (beam envelope). Since spreaders and recombiners were chosen in the horizontal plane, the uniform focusing and lattice regularity was broken in that plane and the horizontal beam envelope requires special attention. On the other hand, the vertical beam size remains small due to maintaining uniform focusing and small beta functions in that plane.

\section{BEAM DYNAMICS CHOICES}

The initial longitudinal acceptance of the linear accelerator $(2.5 \sigma)$ is set by the best performance promised by Ring Coolers. Initial transverse and longitudinal emittaces for Ring Coolers (as they compare to the Straight Cooling Channel) are summarized in Table 1.
TABLE 1. Beam emittance after cooling at $250 \mathrm{MeV} / \mathrm{c}$.

\begin{tabular}{|c|c|c|}
\hline rms values & Cooling Channel & Ring Cooler \\
\hline $\begin{array}{l}\text { Transverse emit- } \\
\text { tance }: \varepsilon_{x}, \varepsilon_{y} \\
\text { (normalized) }\end{array}$ & $2.4 \mathrm{~mm} \cdot \mathrm{nrad}$ & $2.0 \mathrm{~mm} \cdot \mathrm{rad}$ \\
\hline $\begin{array}{l}\text { longitudinal } \\
\text { emittance: } \\
\varepsilon_{\iota}=\sigma_{\Delta \mathrm{p}} \sigma_{\mathrm{z}} / \mathrm{m}_{\mu} \mathrm{c}\end{array}$ & $27 \mathrm{~mm}$ & $3 \mathrm{~mm}$ \\
\hline $\begin{array}{l}\text { momentum } \\
\text { spread: } \sigma_{\Delta \mathrm{p} / \mathrm{p}}\end{array}$ & 0.08 & 0.025 \\
\hline bunch length: $\sigma_{z}$ & $163 \mathrm{~mm}$ & $50 \mathrm{~mm}$ \\
\hline
\end{tabular}

\section{Longitudinal Dynamics}

In the 'Ring Cooler scenario' to perform adiabatic bunching, the RF phase of the cavities needs to be shifted by only 30 deg at the beginning of the preaccelerator and gradually changed to zero by the linac end. In the first half of the linac, when the beam is still not sufficiently relativistic, the offset causes synchrotron motion, allowing bunch compression in both length and momentum spread to $\Delta p / p= \pm 6.5 \%$ and $\Delta \phi= \pm 31 \mathrm{deg}$. The synchrotron motion also suppresses the sag in acceleration for the bunch head and tail. The first frame in Figure 2 shows how the initially elliptical boundary of the bunch longitudinal phase space will be transformed by the end of the linac.

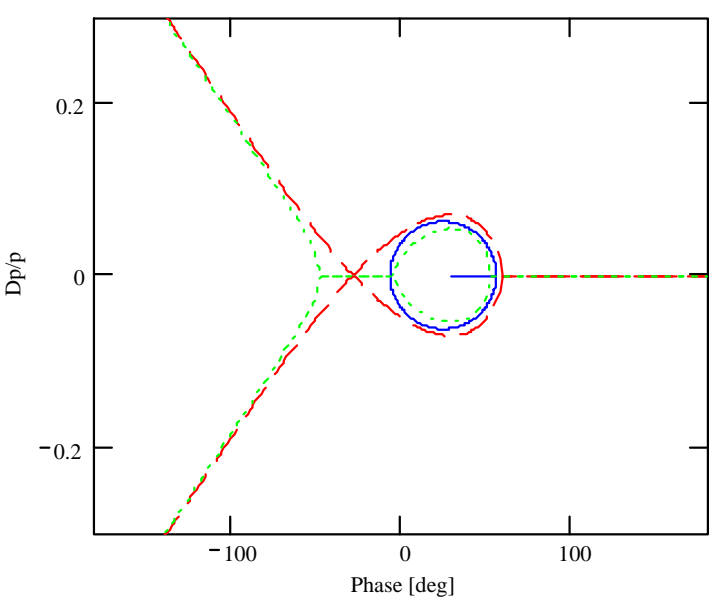

FIGURE 2. Longitudinal beam boundary (solid line) inside a separatrix (dashed line) shown at the beginning of the linac $(\Delta \mathrm{p} / \mathrm{p}= \pm 0.065$ or $\Delta \phi= \pm 31$ deg. $)$ 


\section{Longitudinal Bunch Compression}

Bunch length and energy spread are still too large at the RLA input and their further compression is required in the course of acceleration. To achieve this, the beam is accelerated off-crest with non zero $\mathrm{M}_{56}$. That causes synchrotron motion, which suppresses the longitudinal emittance growth related to non-linearity of accelerating voltage. Without synchrotron motion the minimum beam energy spread would be determined by non-linearity of RF voltage at bunch length and would be equal to $(1-\cos \phi) \approx 6 \%$ for bunch length $\phi=20 \mathrm{deg}$. The synchrotron motion causes particle motion within the bunch and averages the total energy gain of tail's particle to the energy gain of particles in the core. The optimum value is about $90 \mathrm{~cm}$, while optimal detuning of RF phase from on-crest position is different for different arcs. To perform a further bunch compression in 4-pass RLA (see the remaining frames on Figure 3) the beam is again accelerated off-crest with phase offsets in the range of $5-15$ deg for different passes and $\mathrm{M}_{56}$ of $90 \mathrm{~cm}$ for all seven arcs.
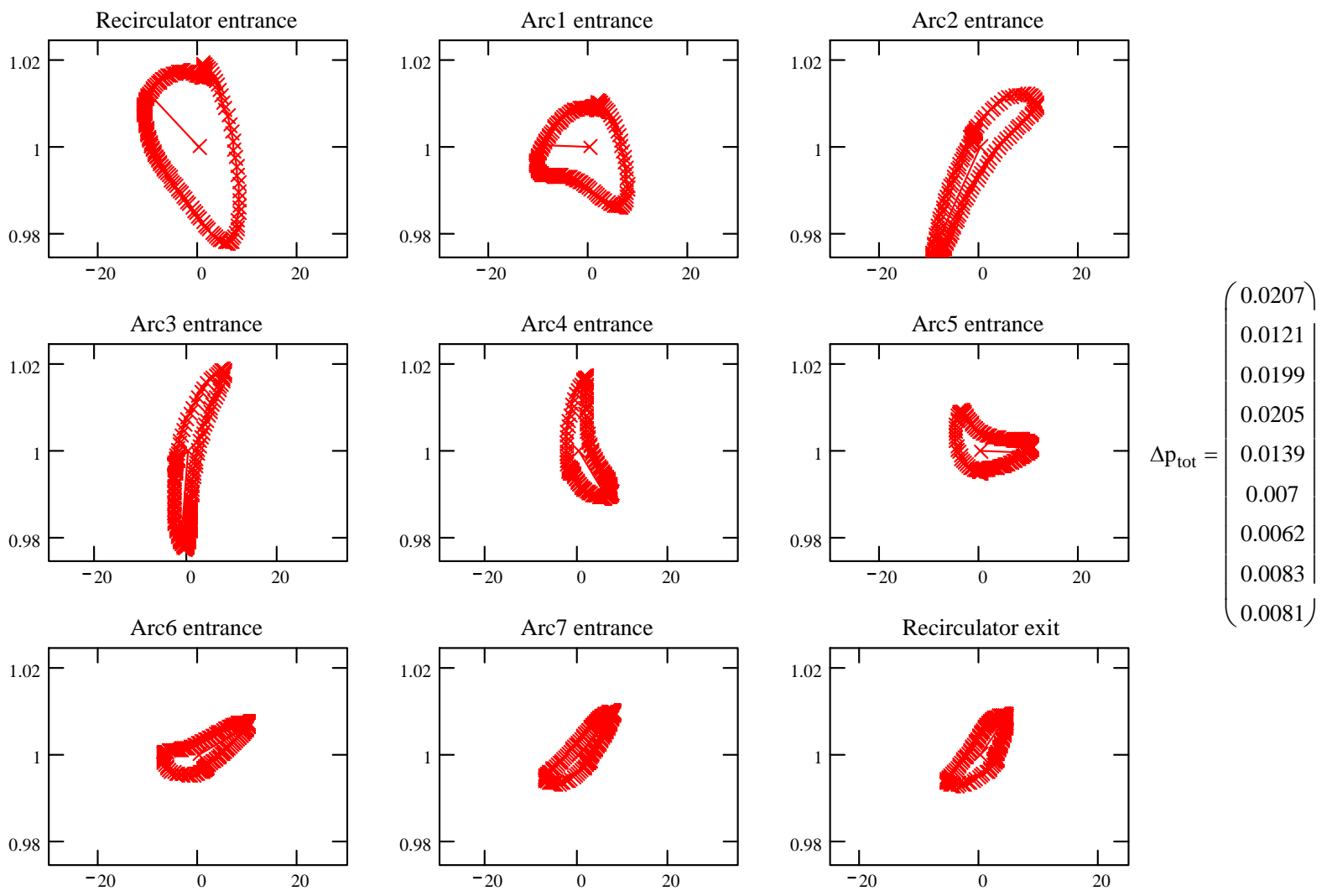

FIGURE 3. Boundaries of the beam longitudinal phase space at different locations in the recirculator; $\mathrm{M}_{56}=90 \mathrm{~cm}$.

\section{Optimized RLA Optics}

To maintain uniform focusing periodicity between linacs and arcs one needs to minimize Twiss functions for multi-pass optics of both linacs. This can be achieved through designing a semi-periodic linac optics (introducing additional large scale lattice period). As illustrated in Figures 4 and 5, resulting Twiss func- tions (at linac ends) are significantly lowered, which facilitates uniform matching between different types of optics to alleviate emittance dilution due to chromatic aberrations. Finally, there is a need for suppression of chromatic effects via sextupole corrections in spreaders and recombiners. This was implemented via three families of sextupoles [2] to control the horizontal emittance blow-up. 
Pass 1

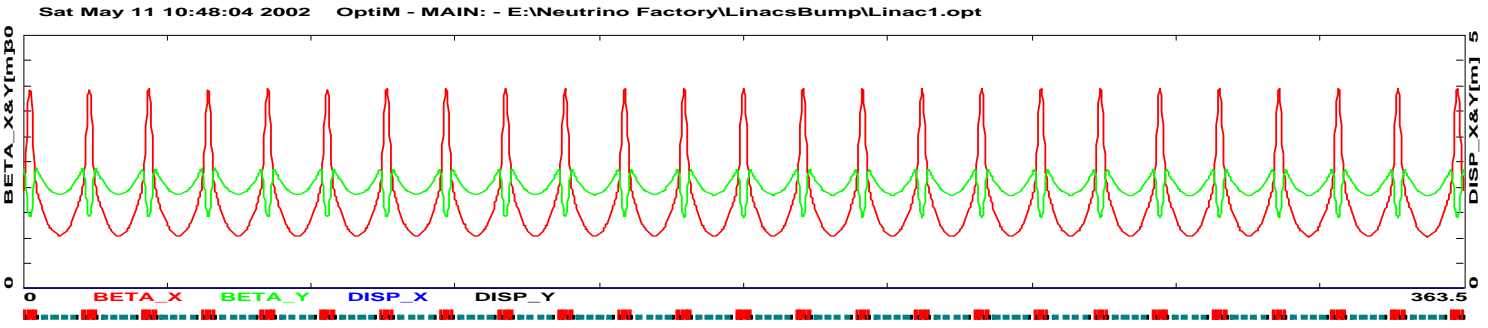

Pass 2

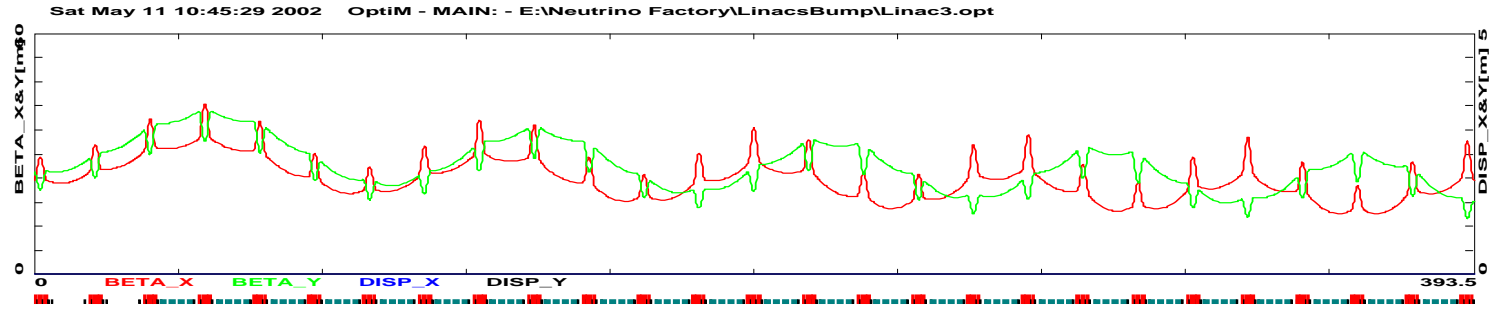

FIGURE 4. Optimized linac optics for multi pass beams - smooth transition Arc-linac.

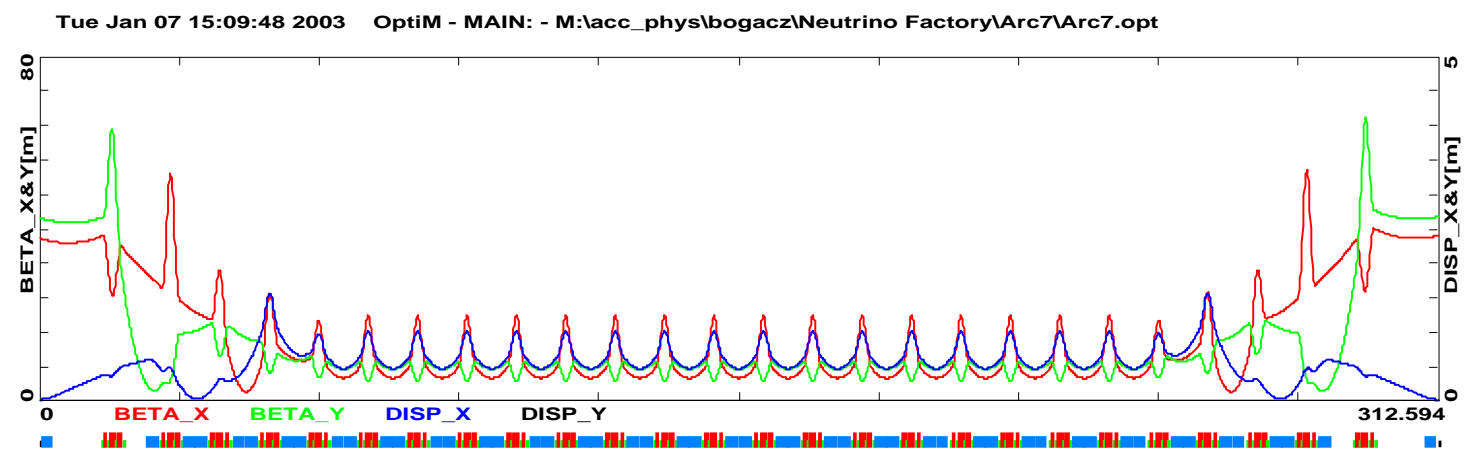

FIGURE 5. Arc 7 Optics - beta-functions and the horizontal dispersion matched to both adjacent linacs.

\section{CONCLUSIONS}

Results of this study suggest that there are no obvious physical or technical limitations precluding construction of an RLA for acceleration of muons to 20 $\mathrm{GeV}$. The proposed acceleration and beam transport scheme is well suited for handling large phase space beams with no emittance dilution [4].

\section{REFERENCES}

1. M.M. Alsharo's et al., Physical Review Special Topics - Accelerators and Beams, Vol 6, 081001 (2003)
2. S.A. Bogacz and V.A. Lebedev, Nuclear Instruments and Methods in Physics Research A, Vol 472/3, 528, (2001)

3. S.A. Bogacz and V.A. Lebedev, Nuclear Instruments and Methods in Physics Research A, Vol 503, 306, (2003)

4. S.A. Bogacz, Journal of Physics G: Nuclear and Particle Physics, Vol 29, 1723 (2003) 